\title{
ON A WEAK FORM OF WEAK QUASI-CONTINUITY
}

\author{
C. W. BAKER
}

Received 12 September 2001

\begin{abstract}
A weak form of weak quasi-continuity, which we call subweak quasi-continuity, is introduced. It is shown that subweak quasi-continuity is strictly weaker than weak quasicontinuity. Subweak quasi-continuity is used to strengthen several results in the literature concerning weak quasi-continuity. Specifically, results concerning the graph, graph function, and restriction of a weakly quasi-continuous function are extended slightly. Also, a result concerning weakly quasi-continuous retractions is strengthened.
\end{abstract}

2000 Mathematics Subject Classification: 54C10.

1. Introduction. Weakly quasi-continuous functions were introduced by Popa and Stan [9]. Recently, weak quasi-continuity has been developed further by Noiri [5, 6] and Park and Ha [8]. Due to a result by Noiri [5], weak quasi-continuity is equivalent to the weak semicontinuity developed by Arya and Bhamini [1]. The purpose of this note is to introduce the concept of subweak quasi-continuity, which we define in terms of a base for the topology on the codomain. We establish that this condition is strictly weaker than weak quasi-continuity and we use it to strengthen some of the results in the literature concerning weak quasi-continuity. For example, we show that the graph of a subweakly quasi-continuous function with a Hausdorff codomain is semiclosed. We also show that, if the graph function is subweakly quasi-continuous with respect to the usual base for the product space, then the function itself is weakly quasi-continuous, and that, if a function is subweakly quasi-continuous with respect to the base $\mathscr{B}$, then the restriction to a preopen set is subweakly quasi-continuous with respect to the same base. These results strengthen slightly the comparable results for weakly quasi-continuous functions. Finally, we extend a result concerning weakly quasi-continuous retractions and investigate some of the basic properties of subweakly quasi-continuous functions.

2. Preliminaries. The symbols $X$ and $Y$ denote topological spaces with no separation axioms assumed unless explicitly stated. All sets are considered to be subsets of topological spaces. The closure and interior of a set $A$ are signified by $\mathrm{Cl}(A)$ and $\operatorname{Int}(A)$, respectively. A set $A$ is semiopen (preopen, $\alpha$-open) provided that $A \subseteq \mathrm{Cl}(\operatorname{Int}(A))(A \subseteq$ $\operatorname{Int}(\mathrm{Cl}(A)), A \subseteq \operatorname{Int}(\mathrm{Cl}(\operatorname{Int}(A))))$. A set is semiclosed (preclosed, $\alpha$-closed) provided that its complement is semiopen (preopen, $\alpha$-open). The collection of all semiopen sets in a space $X$ is denoted by $\mathrm{SO}(X)$ and the collection of all semiopen sets in $X$ containing a fixed point $x$ is denoted by $\mathrm{SO}(X, x)$. The intersection of all semiclosed sets containing a set $A$ is called the semiclosure of $A$ and denoted by $\operatorname{sCl}(A)$. The 
semi-interior of a set $A$, denoted by $\operatorname{sint}(A)$, is the union of all semiopen sets contained in $A$. The preclosure of $A$, denoted by $\mathrm{pCl}(A)$, is the intersection of all preclosed sets containing $A$. Finally, if an operator is used with respect to a proper subspace, a subscript is added to the operator. Otherwise, it is assumed that the operator refers to the entire space.

Definition 2.1 (Popa and Stan [9]). A function $f: X \rightarrow Y$ is said to be weakly quasi-continuous if for every $x \in X$, every open set $U$ in $X$ containing $x$, and every open set $V$ in $Y$ containing $f(x)$, there exists a nonempty open set $W$ in $X$ such that $W \subseteq U$ and $f(W) \subseteq \mathrm{Cl}(V)$.

DefinItion 2.2 (Arya and Bhamini [1]). A function $f: X \rightarrow Y$ is said to be weakly semicontinuous if for every $x \in X$ and every open set $V$ in $Y$ containing $f(x)$, there exists $U \in \mathrm{SO}(X, x)$ for which $f(U) \subseteq \mathrm{Cl}(V)$.

The following result by Noiri [5] shows that weak quasi-continuity and weak semicontinuity are equivalent.

Theorem 2.3 (Noiri [5, Theorem 4.1]). A function $f: X \rightarrow Y$ is weakly quasi-continuous if and only if for every $x \in X$ and every open set $V$ containing $f(x)$, there exists $U \in \mathrm{SO}(X, x)$ for which $f(U) \subseteq \mathrm{Cl}(V)$.

DEFINITION 2.4. A function $f: X \rightarrow Y$ is said to be subweakly continuous (Rose [10]) (subalmost weakly continuous (Baker [2])) if there is an open base $\mathscr{B}$ for the topology on $Y$ such that $\mathrm{Cl}\left(f^{-1}(V)\right) \subseteq f^{-1}(\mathrm{Cl}(V))\left(\mathrm{pCl}\left(f^{-1}(V)\right) \subseteq f^{-1}(\mathrm{Cl}(V))\right)$ for every $V \in \mathscr{B}$.

3. Subweakly quasi-continuous functions. The following characterization of weak quasi-continuity is due to Noiri [5].

TheOrem 3.1 (Noiri [5, Theorem 4.3(d)]). A function $f: X \rightarrow Y$ is weakly quasicontinuous if and only if $\mathrm{sCl}\left(f^{-1}(V)\right) \subseteq f^{-1}(\mathrm{Cl}(V))$ for every open set $V$ in $Y$.

We define a function $f: X \rightarrow Y$ to be subweakly quasi-continuous provided that there is an open base $\mathscr{B}$ for the topology on $Y$ for which $\operatorname{sCl}\left(f^{-1}(V)\right) \subseteq f^{-1}(\mathrm{Cl}(V))$ for every $V \in \mathscr{B}$. Obviously, weak quasi-continuity implies subweak quasi-continuity. The following example shows that these concepts are not equivalent.

EXAMPLE 3.2. Let $X=\mathbb{R}$ have the usual topology and $Y=X$ have the discrete topology. The identity mapping $f: X \rightarrow Y$ is subweakly quasi-continuous with respect to the base consisting of the singleton sets in $Y$. However, $f$ is not weakly quasicontinuous because for $V=(0,1) \cup(1,2), \operatorname{sCl}\left(f^{-1}(V)\right) \nsubseteq f^{-1}(\mathrm{Cl}(V))$.

Since $\operatorname{sCl}(A)=A \cup \operatorname{Int}(\mathrm{Cl}(A))$ for every set $A$, we have the following characterization of subweak quasi-continuity.

THEOREM 3.3. A function $f: X \rightarrow Y$ is subweakly quasi-continuous if and only if there is an open base $\mathscr{B}$ for the topology on $Y$ for which $\operatorname{Int}\left(\mathrm{Cl}\left(f^{-1}(V)\right)\right) \subseteq f^{-1}(\mathrm{Cl}(V))$ for every $V \subseteq \mathscr{B}$. 
Since $\mathrm{sCl}(A) \subseteq \mathrm{Cl}(A)$ for every set $A$, obviously, subweak continuity implies subweak quasi-continuity. The following example shows that the converse implication does not hold.

EXAMPLE 3.4. Let $X=[1 / 2,3 / 2]$ have the usual relative topology, $Y=\{0,1\}$ have the discrete topology, and let $f: X \rightarrow Y$ be the greatest integer function. Kar and Bhattacharya [3] showed that $f$ is weakly quasi-continuous (their term is weakly semicontinuous) but not weakly continuous. Obviously, the function $f$ is also not subweakly continuous.

The following two examples establish that subweak quasi-continuity is independent of subalmost weak continuity.

EXAMPLE 3.5. Let $X$ be an indiscrete space with at least two elements and let $Y=X$ have the discrete topology. Since $\operatorname{pCl}(\{x\})=\{x\}$ for every $x \in X$, the identity mapping $f: X \rightarrow Y$ is subalmost weakly continuous with respect to the base consisting of the singleton sets in $Y$. However, since singleton sets in $X$ are dense, $f$ is not subweakly quasi-continuous.

EXAMPLE 3.6. Let $X=\{a, b, c\}$ have the topology $\tau=\{X, \varnothing,\{a\},\{b\},\{a, b\}\}$ and $Y=X$ have the discrete topology. Let $f: X \rightarrow Y$ be the identity mapping. The function $f$ is not subalmost weakly continuous, since any base for $Y$ must include $V=\{a\}$ and $\mathrm{pCl}\left(f^{-1}(V)\right) \nsubseteq f^{-1}(\mathrm{Cl}(V))$. However, $f$ is subweakly quasi-continuous with respect to the base of singleton subsets of $Y$.

4. Graph related properties. Recall that the graph of a function $f: X \rightarrow Y$ is the subspace $G(f)=\{(x, f(x)): x \in X\}$ of the product space $X \times Y$.

Park and Ha [8] proved that the graph of a weakly quasi-continuous function with a Hausdorff codomain is semiclosed. We show that weak quasi-continuity can be replaced by subweak quasi-continuity.

THEOREM 4.1. If $f: X \rightarrow Y$ is subweakly quasi-continuous and $Y$ is Hausdorff, then the graph of $f, G(f)$, is semiclosed.

Proof. Let $\mathscr{B}$ be an open base for $Y$ such that $\mathrm{sCl}\left(f^{-1}(V)\right) \subseteq f^{-1}(\mathrm{Cl}(V))$ for every $V \in \mathscr{B}$. Let $(x, y) \in X \times Y-G(f)$. Since $y \neq f(x)$, there exists disjoint open sets $V$ and $W$ with $f(x) \in W, y \in V$, and $V \in \mathscr{B}$. Then $x \notin f^{-1}(\mathrm{Cl}(V))$, and, since $\operatorname{sCl}\left(f^{-1}(V)\right) \subseteq$ $f^{-1}(\mathrm{Cl}(V)), x \notin \mathrm{sCl}\left(f^{-1}(V)\right)$. Therefore $(x, y) \in\left(X-\operatorname{sCl}\left(f^{-1}(V)\right)\right) \times V \subseteq X \times Y-G(f)$. Since $\mathrm{sCl}\left(f^{-1}(V)\right)$ is semiclosed, $X-\operatorname{sCl}\left(f^{-1}(V)\right)$ is semiopen. Since finite products of semiopen sets are semiopen, $\left(X-\mathrm{sCl}\left(f^{-1}(V)\right)\right) \times V$ is semiopen. Finally, since unions of semiopen sets are semiopen, it follows that $X \times Y-G(f)$ is semiopen and that $G(f)$ is semiclosed.

COROLlary 4.2 (Park and Ha [8, Corollary 4.2]). If $f: X \rightarrow Y$ is weakly quasicontinuous and $Y$ is Hausdorff, then the graph of $f, G(f)$, is semiclosed.

By the graph function of a function $f: X \rightarrow Y$ we mean the function $g: X \rightarrow X \times Y$ given by $g(x)=(x, f(x))$ for every $x \in X$. 
THEOREM 4.3. Let $f:(X, \tau) \rightarrow(Y, \sigma)$ be a function and let $\mathscr{B}$ be an open base for $\sigma$. Let $\mathscr{C}=\{U \times V: U \in \tau, V \in \mathscr{B}\}$. The function $f$ is subweakly quasi-continuous with respect to the base $\mathscr{B}$ if and only if the graph function of $f, g: X \rightarrow X \times Y$, is subweakly quasi-continuous with respect to the base $\mathscr{C}$.

Proof. Assume that $f:(X, \tau) \rightarrow(Y, \sigma)$ is subweakly quasi-continuous with respect to the base $\mathscr{B}$ for $\sigma$. Let $U \times V \in \mathscr{C}$, where $U \in \tau$ and $V \in \mathscr{B}$. Then $\operatorname{sCl}\left(g^{-1}(U \times\right.$ $V))=\operatorname{sCl}\left(U \cap f^{-1}(V)\right) \subseteq \operatorname{sCl}(U) \cap \operatorname{sCl}\left(f^{-1}(V)\right) \subseteq \mathrm{Cl}(U) \cap f^{-1}(\mathrm{Cl}(V))=g^{-1}(\mathrm{Cl}(U) \times$ $\mathrm{Cl}(V))=g^{-1}(\mathrm{Cl}(U \times V))$. Thus $g$ is subweakly quasi-continuous with respect to the base $\mathscr{C}$.

Assume that $g:(X, \tau) \rightarrow X \times Y$ is subweakly quasi-continuous with respect to the base $\mathscr{C}$ for $X \times Y$. If $V \in \mathscr{B}$, then $\operatorname{sCl}\left(f^{-1}(V)\right)=\operatorname{sCl}\left(g^{-1}(X \times V)\right) \subseteq g^{-1}(\mathrm{Cl}(X \times V))=$ $g^{-1}(X \times \mathrm{Cl}(V))=f^{-1}(\mathrm{Cl}(V))$. Therefore, $f$ is subweakly quasi-continuous with respect to the base $\mathscr{B}$.

In Theorem 4.3, if we take $\mathscr{B}$ to be $\sigma$, the topology on $Y$, then we have the following result.

COROLLARY 4.4. If the graph function $g: X \rightarrow X \times Y$ of a function $f$ is subweakly quasi-continuous with respect to the usual base for the product space, then the function $f$ is weakly quasi-continuous.

COROLLARY 4.5 (Noiri [5, The "only if" part of Theorem 6.3.4]). If the graph function $g: X \rightarrow X \times Y$ of a function $f$ is weakly quasi-continuous, then the function $f$ is weakly quasi-continuous.

\section{Additional properties}

DEFINITION 5.1 (Kar and Bhattacharya [4]). A space $X$ is said to be semi- $T_{1}$ provided that for every pair of distinct points $x$ and $y$ in $X$ there exist sets $U \in \operatorname{SO}(X, x)$ and $V \in \mathrm{SO}(X, y)$ such that $y \notin U$ and $x \notin V$.

THEOREM 5.2. If $Y$ is Hausdorff and $f: X \rightarrow Y$ is a subweakly quasi-continuous injection, then $X$ is semi- $T_{1}$.

Proof. Let $x_{1}$ and $x_{2}$ be distinct points in $X$ and let $\mathscr{B}$ be an open base for $Y$ such that $\operatorname{sCl}\left(f^{-1}(V)\right) \subseteq f^{-1}(\mathrm{Cl}(V))$ for every $V \in \mathscr{B}$. Since $Y$ is Hausdorff and $f\left(x_{1}\right) \neq$ $f\left(x_{2}\right)$, there exist disjoint open sets $U$ and $V$ in $Y$ with $f\left(x_{1}\right) \in U$ and $f\left(x_{2}\right) \in V$, and $V \in \mathscr{B}$. Then, since $f\left(x_{1}\right) \notin \mathrm{Cl}(V)$, we have $x_{1} \in X-f^{-1}(\mathrm{Cl}(V)) \subseteq X-\operatorname{sCl}\left(f^{-1}(V)\right)$ which is semiopen and does not contain $x_{2}$. Therefore $X$ is semi- $T_{1}$.

The function in Example 3.6 is a subweakly quasi-continuous injection with a Hausdorff codomain and a non- $T_{1}$-domain. Therefore, the conclusion that $X$ is semi- $T_{1}$ in Theorem 5.2 cannot be strengthened to $T_{1}$.

Since the restriction of the function $f$ in Example 3.6 to the set $A=\{a, c\}$ is not subweakly quasi-continuous, we see that the restriction of a subweakly quasi-continuous function can fail to be subweakly quasi-continuous. Noiri [5] proved that the restriction of weakly quasi-continuous function to an open set is weakly quasi-continuous and Arya and Bhamini [1] extended this result to $\alpha$-open sets. Finally, Park and Ha [8] 
extended the result further to preopen sets. In what follows, we establish the analogous result for subweakly quasi-continuous functions.

THEOREM 5.3. If $f: X \rightarrow Y$ is subweakly quasi-continuous with respect to the base $\mathscr{B}$ for $Y$ and $A$ is a preopen set in $X$, then $\left.f\right|_{A}: A \rightarrow Y$ is subweakly quasi-continuous with respect to the base $\mathscr{B}$.

Proof. Let $V \in \mathscr{B}$, then using (Noiri [7, Lemma 3.3]) we see that $\operatorname{sCl}_{A}\left(\left.f\right|_{A} ^{-1}(V)\right)=$ $A \cap \operatorname{sCl}\left(\left.f\right|_{A} ^{-1}(V)\right)=A \cap \operatorname{sCl}\left(f^{-1}(V) \cap A\right) \subseteq A \cap \operatorname{sCl}\left(f^{-1}(V)\right) \subseteq A \cap f^{-1}(\mathrm{Cl}(V))=$ $\left.f\right|_{A} ^{-1}(\mathrm{Cl}(V))$. Therefore, $\left.f\right|_{A}: A \rightarrow Y$ is subweakly quasi-continuous with respect to the base $\mathscr{B}$.

In Theorem 5.3, if we let $\mathscr{B}$ be the topology, then we have the following result.

COROLlary 5.4 (Park and Ha [8, Theorem 3.8]). If $f: X \rightarrow Y$ is weakly quasicontinuous and $A$ is an preopen set in $X$, then $\left.f\right|_{A}: A \rightarrow Y$ is weakly quasi-continuous.

THEOREM 5.5. If $f: X \rightarrow Y$ is subweakly quasi-continuous and $A$ is an open set in $Y$ containing $f(X)$, then $f: X \rightarrow A$ is subweakly quasi-continuous.

Proof. Let $\mathscr{B}$ be an open base for $Y$ for which $\mathrm{sCl}\left(f^{-1}(V)\right) \subseteq f^{-1}(\mathrm{Cl}(V))$ for every $V \in \mathscr{B}$. Then $\mathscr{C}=\{V \cap A: V \in \mathscr{B}\}$ is an open base for the relative topology on $A$. Let $V \cap A \in \mathscr{C}$, where $V \in \mathscr{B}$. Then $\operatorname{sCl}\left(f^{-1}(V \cap A)\right)=\operatorname{sCl}\left(f^{-1}(V)\right) \subseteq f^{-1}(\mathrm{Cl}(V))=$ $f^{-1}(\mathrm{Cl}(V) \cap A)$. The proof is completed by establishing that $\mathrm{Cl}(V) \cap A \subseteq \mathrm{Cl}_{A}(V \cap A)$.

Let $y \in \mathrm{Cl}(V) \cap A$ and let $W \subseteq A$ be open in $A$ with $y \in W$. Since $A$ is open in $Y, W$ is open in $Y$. Because $y \in \mathrm{Cl}(V), W \cap V \neq \varnothing$. Therefore $W \cap(V \cap A) \neq \varnothing$, which proves that $y \in \mathrm{Cl}_{A}(V \cap A)$. Thus $\mathrm{Cl}(V) \cap A \subseteq \mathrm{Cl}_{A}(V \cap A)$.

Now, it follows that $f: X \rightarrow A$ is subweakly quasi-continuous.

Park and Ha [8] defined a function $f: X \rightarrow A$, where $A \subseteq X$, to be a weakly quasicontinuous retraction provided that $f$ is weakly quasi-continuous and $\left.f\right|_{A}$ is the identity on $A$. It is then proved (Park and Ha [8, Theorem 3.15]) that, if $f: X \rightarrow A$ is a weakly quasi-continuous retraction and $X$ is Hausdorff, then $A$ is semiclosed in $X$. We prove the following comparable result for subweakly quasi-continuous functions.

THEOREM 5.6. Let $A \subseteq X$ and let $f: X \rightarrow X$ be a subweakly quasi-continuous function such that $f(X)=A$ and $\left.f\right|_{A}$ is the identity on A. If $X$ is Hausdorff, then $A$ is semiclosed.

Proof. Assume $A$ is not semiclosed. Let $x \in \operatorname{sCl}(A)-A$. Let $\mathscr{B}$ be an open base for the topology on $X$ such that $\mathrm{sCl}\left(f^{-1}(V)\right) \subseteq f^{-1}(\mathrm{Cl}(V))$ for every $V \in \mathscr{B}$. Since $x \notin A$, $x \neq f(x)$. Because $X$ is Hausdorff, there exist disjoint open sets $V$ and $W$ such that $x \in V, f(x) \in W$, and $V \in \mathscr{B}$. Let $U \in \mathrm{SO}(X, x)$. Then $x \in U \cap V$, which is semiopen in $X$ (Noiri [7]). Since $x \in \operatorname{sCl}(A),(U \cap V) \cap A \neq \varnothing$. So there exists $y \in(U \cap V) \cap A$. Since $y \in A, f(y)=y$ and therefore $y \in f^{-1}(V)$. Thus $U \cap f^{-1}(V) \neq \varnothing$ and we see that $x \in \operatorname{sCl}\left(f^{-1}(V)\right)$. However, $f(x) \in W$, which is open and disjoint from $V$. Hence $f(x) \notin \mathrm{Cl}(V)$ or $x \notin f^{-1}(\mathrm{Cl}(V))$, which contradicts the assumption that $f$ is subweakly quasi-continuous.

LEMмA 5.7. If $A \subseteq Y$ and $f: X \rightarrow A$ is weakly quasi-continuous, then $f: X \rightarrow Y$ is weakly quasi-continuous. 
Proof. If $V$ is an open set in $Y$, then $\mathrm{sCl}\left(f^{-1}(V)\right)=\mathrm{sCl}\left(f^{-1}(V \cap A)\right) \subseteq f^{-1}\left(\mathrm{Cl}_{A}(V \cap\right.$ $A))=f^{-1}(A \cap \mathrm{Cl}(V \cap A))=f^{-1}(\mathrm{Cl}(V \cap A)) \subseteq f^{-1}(\mathrm{Cl}(V))$.

Thus a weakly quasi-continuous retraction satisfies the hypothesis of Theorem 5.6 and we have the following corollary.

Corollary 5.8 (Park and Ha [8, Theorem 3.15]). If $f: X \rightarrow A$, where $A \subseteq X$, is a weakly quasi-continuous retraction and $X$ is Hausdorff, then $A$ is semiclosed.

THeOrem 5.9. Let $Y$ be a Hausdorff space, $f_{1}: X \rightarrow Y$ continuous, and $f_{2}: X \rightarrow Y$ subweakly quasi-continuous. Then $\left\{x \in X: f_{1}(x)=f_{2}(x)\right\}$ is semiclosed.

Proof. Let $A=\left\{x \in X: f_{1}(x)=f_{2}(x)\right\}$ and let $x \in X-A$. Let $\mathscr{B}$ be an open base for the topology on $Y$ for which $\operatorname{sCl}\left(f_{2}^{-1}(V)\right) \subseteq f_{2}^{-1}(\mathrm{Cl}(V))$ for every $V \in \mathscr{B}$. Since $Y$ is Hausdorff and $f_{1}(x) \neq f_{2}(x)$, there exist disjoint open sets $V$ and $W$ in $Y$ for which $f_{1}(x) \in V, f_{2}(x) \in W$, and $V \in \mathscr{B}$. Since $f_{2}(x) \notin \mathrm{Cl}(V)$, we have $x \in X-f_{2}^{-1}(\mathrm{Cl}(V)) \subseteq$ $X-\operatorname{sCl}\left(f_{2}(V)\right)$. Therefore $x \in f_{1}^{-1}(V) \cap\left(X-\operatorname{sCl}\left(f_{2}^{-1}(V)\right)\right) \subseteq X-A$. Since $f_{1}^{-1}(V)$ is open, $X-\mathrm{sCl}\left(f_{2}^{-1}(V)\right)$ is semiopen, and the intersection of an open set and a semiopen set is semiopen (Noiri [7]), we see that $X-A$ is semiopen and that $A$ is semiclosed.

Corollary 5.10. Let $Y$ be Hausdorff, $f_{1}: X \rightarrow Y$ continuous, and $f_{2}: X \rightarrow Y$ subweakly quasi-continuous. If $f_{1}$ and $f_{2}$ agree on a dense subset of $X$, then $f_{1}=f_{2}$.

ACKNOWLEDGMENT. The author gratefully acknowledges the support of Indiana University Southeast in the publication of this paper.

\section{REFERENCES}

[1] S. P. Arya and M. P. Bhamini, Some weaker forms of semicontinuous functions, Ganita 33 (1982), no. 1-2, 124-134.

[2] C. W. Baker, On a weak form of almost weakly continuous functions, Demonstratio Math. 33 (2000), no. 4, 865-872.

[3] A. Kar and P. Bhattacharya, Weakly semicontinuous functions, J. Indian Acad. Math. 8 (1986), no. 2, 83-93.

[4] Some weak separation axioms, Bull. Calcutta Math. Soc. 82 (1990), no. 5, 415-422.

[5] T. Noiri, Properties of some weak forms of continuity, Int. J. Math. Math. Sci. 10 (1987), no. $1,97-111$.

[6] Weakly $\alpha$-continuous functions, Int. J. Math. Math. Sci. 10 (1987), no. 3, 483-490.

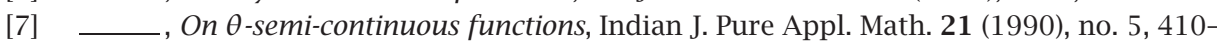
415.

[8] J. H. Park and H. Y. Ha, A note on weakly quasi-continuous functions, Int. J. Math. Math. Sci. 19 (1996), no. 4, 767-772.

[9] V. Popa and C. Stan, On a decomposition of quasi-continuity in topological spaces, Stud. Cerc. Mat. 25 (1973), 41-43 (Romanian).

[10] D. A. Rose, Weak continuity and almost continuity, Int. J. Math. Math. Sci. 7 (1984), no. 2, 311-318.

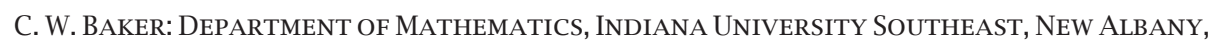
IN 47150, USA

E-mail address: cbaker@ius.edu 


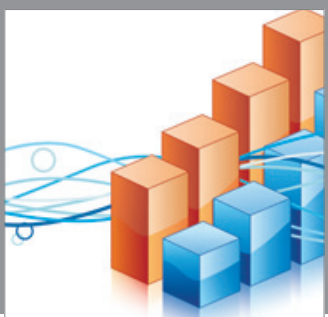

Advances in

Operations Research

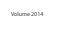

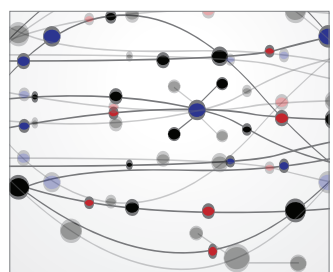

\section{The Scientific} World Journal
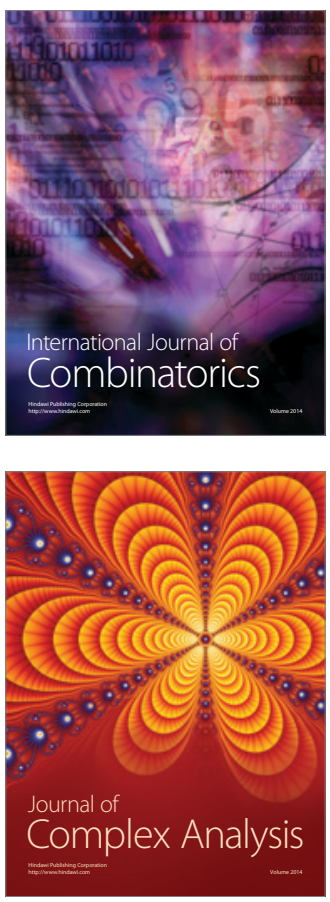

International Journal of

Mathematics and

Mathematical

Sciences
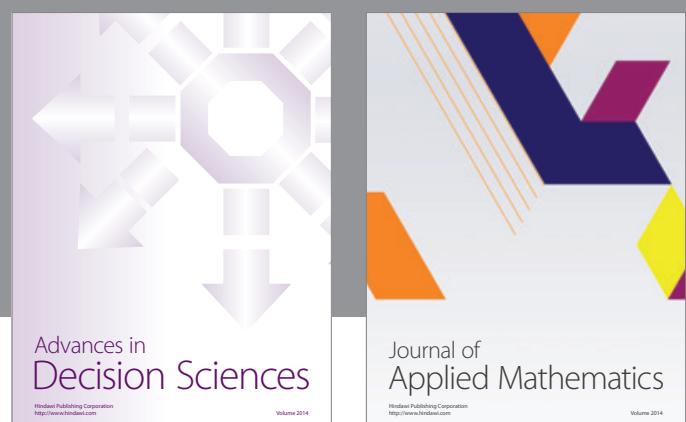

Journal of

Applied Mathematics
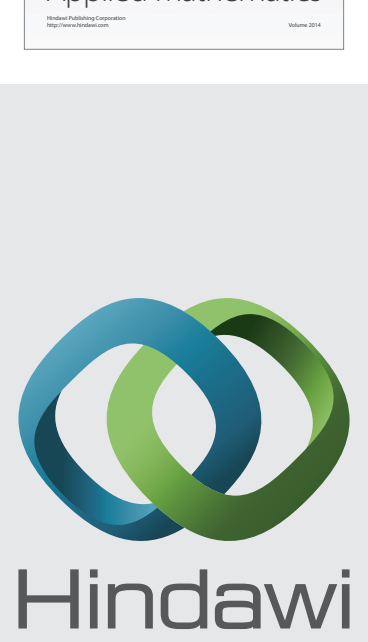

Submit your manuscripts at http://www.hindawi.com
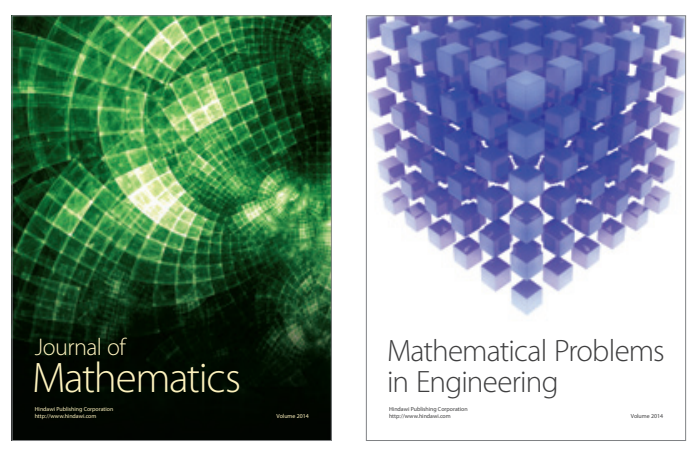

Mathematical Problems in Engineering
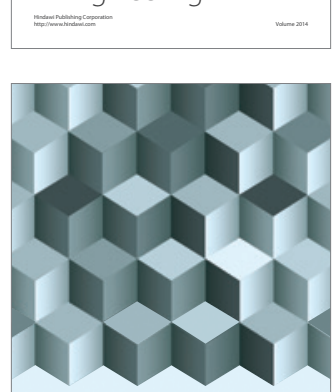

Journal of

Function Spaces
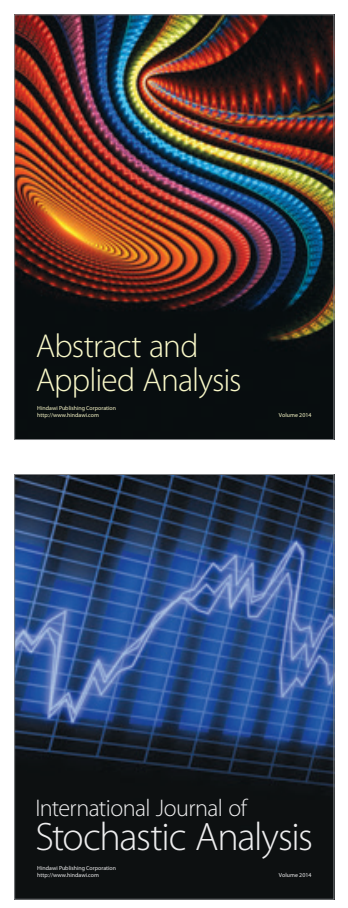

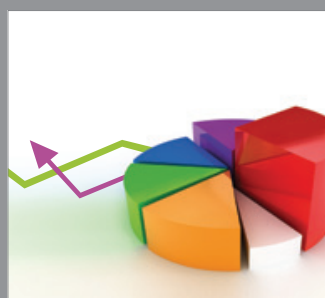

ournal of

Probability and Statistics

Promensencen
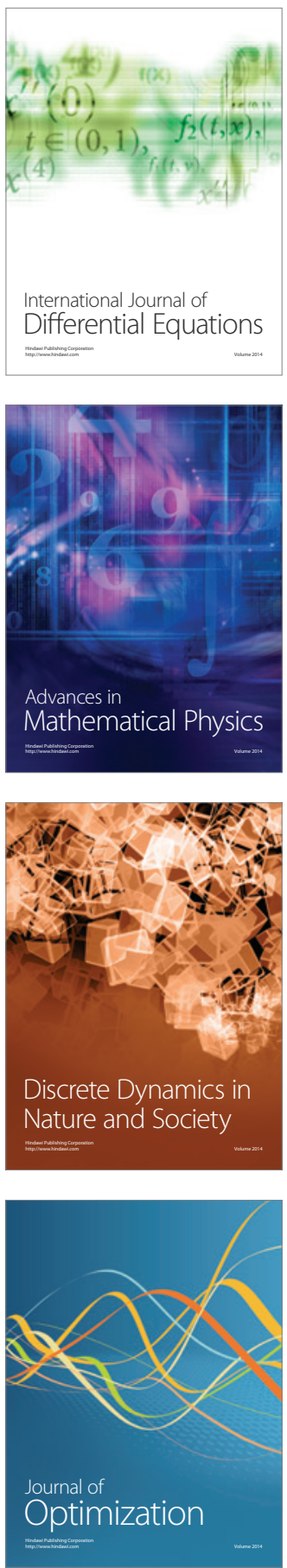